\title{
Désir et Addiction
}

\author{
Pascal COLAS
}

Médecin Sexologue

Président du Collège Lyonnais de Sexologie

\section{RÉSUMÉ}

Analysant l'étymologie des deux termes, désir et addiction, un discernement s'impose entre ce qui les unit et ce qui les oppose.

Le désir est une prise de conscience d'une tendance qui porte à vouloir obtenir un objet connu ou imaginaire.

L'addiction est un emprunt à la langue anglaise to addict : s'adonner à.... Dérivé du mot latin addictere qui signifiait : mise en esclavage, aliénation, contrainte par corps.

L'immobilisation observée dans l'aliénation inhérente à l'addiction s'oppose à la tendance, prise en tant que mouvement, incombant au désir. Par là-même, le désir pourrait être d'essence active, l'addiction d'essence passive. Le désir émanation de la vie, l'addiction expression d'un processus mortifère.

Le désir amoureux pourrait aussi être éloigné de la dépendance amoureuse (addiction), et la phrase utu me manques " comme une interrogation sur la dimension addictive du lien amoureux.

Mots clés : alcool, addiction, toxicomanie, désir, amour, alexithymie

\section{INTRODUCTION}

Analysant l'étymologie des deux termes, désir et addiction, un discernement s'impose entre ce qui les unit, les lie et ce qui les oppose, les éloigne.

Le mot désir dérive du verbe désirer qui lui est préséant. On lui attribue deux définitions [9]:

1) prise de conscience d'une tendance particulière qui porte à vouloir obtenir un objet connu ou imaginaire.
2) tendance consciente aux plaisirs sexuels.

Le mot addiction lui, est un emprunt à la langue anglaise to addict : s'adonner à... Anglicisme lui-même dérivé du latin addictere qui signifiait : mise en esclavage, aliénation, contrainte par corps.

L'immobilisation observée dans l'aliénation inhérente à l'addiction s'oppose à la tendance, prise en tant que mouvement, incombant au désir. Par là même, le désir pourrait être d'essence active, l'addiction d'essence passive [4]. Le désir émanation de la vie, l'addiction expression d'un processus mortifère. Le couple désir et addiction alors assimilable à la représentation d'Eros et Thanatos [11].

\section{L'ALEXITHYMIE}

L'addiction est caractérisée en premier lieu par la dépendance. Même si tout un chacun vit en dépendance avec son environnement (air, oxygène, tissu social...), ce qui est remarquable dans l'addiction c'est avant tout la création de dépendance à des produits ou à des conduites nonnécessaires biologiquement ou socialement. Les plus classiques comprennent l'alcoolisme, l'usage de psychotropes (drogues ou médicaments), le tabagisme... D'autres addictions plus récentes viennent toutefois en compléter la liste : le jeu, la conduite à risque, internet, et pourquoi pas l'accroche au voyeurisme du banal par certains reality-shows.

La dépendance a pour corollaires le manque et le syndrôme de sevrage attenant dans certains cas. La perte de contrôle vis-à-vis du produit ou de la conduite, avec besoin impérieux (et non désir ou envie) et répétition, accompagnent l'aspect addictif de la consommation.

Correspondance :

Dr Pascal COLAS - 28 rue Franklin, 69002 LYON Email pascolas@tiscali.fr 
On assiste alors à la mise en place d'une réelle économie du mode de vie dans laquelle tout est organisé autour du produit (ex : réserve par peur du manque).

L'addiction ne peut en aucun cas être considérée comme un symptôme typant une structure de personnalité, mais plutôt analysée comme un comportement. Sa genèse se ferait vraisemblablement très tôt dans le développement de la personnalité, bien avant l'acquisition du langage. Dès le stade oral. Ne dit-on pas de l'alcoolique qu'il biberonne?

Pour expliquer l'origine du comportement addictif la notion d'alexithymie peut être retenue. C'est en tout cas ce que la psychanalyste Joyce McDougall propose au sujet de l'alcoolisme [7].

En soutenant que c'est l'indexation affective des expériences du nourrisson qui donne le sens, on sous-entend que c'est la mère et ses attitudes qui vont être génératrices du sens pour l'enfant.

Dans l'alexithymie (impossibilité de mettre un mot sur un affect), les expériences du nourrisson sont vécues sans sens. Un exemple malheureusement trop répandu peut éclairer ces propos. II s'agit des " bébés théralène ou valium " ou des " bébés tétines ". Bébé pleure. Bon, soit il a faim, soit il a soif, soit il a des coliques, soit il a une douleur.... Si la réponse de la mère est systématiquement et sans discernement de donner la tétine (bouche trou ?) ou un médicament calmant pour apaiser les pleurs intempestifs, aucun sens ne sera donné (par la mère) à la souffrance de l'enfant. La réponse aux pleurs est littéralement insensée. Réponse au besoin non symbolisé car non compris par la mère.

On aboutirait ainsi à une sorte de clivage de la personnalité où une partie de Moi serait défaillante. Sorte de zone d'ombre, pièce manquante ou maille sautée du tricot [5]. Sorte de trou qui ferait que la béance serait insatiable, jamais satisfaite et devrait en permanence être remplie par un produit, une drogue ou une conduite.... voire une personne.

II s'agit donc avant tout d'un besoin de remplissage...[6] bien éloigné donc de la dynamique du désir. Si le désir se nourrit du sens et fait appel à la fonction symbolique, l'addiction elle n'est qu'une réponse insensée à ce trou.

Du reste, on dit bien de l'alcoolique qu'il " boit comme un trou ».

\section{L'HOMME FRIGORIFIÉ}

L'alcoolique, par bien des aspects pourrait être considéré, comme Bukowski le fait de lui-même comme " un homme frigorifié " [2]. Dans Mémoire d'un vieux dégueulasse, il dresse son auto-portrait : je suis devenu « insensible... l'esprit vide, ni heureux, ni malheureux. L'indifférence est ma marque de fabrique. Je me FOUS de tout, je ne me mets jamais en colère, la haine, l'espoir et le bonheur me sont totalement inconnus ". Cet autoportrait décrit, de manière éloquente, combien le désir s'est totalement évaporé de la dynamique du sujet alcoolique [10].
Mais l'attitude face à l'alcool est fonction de chacun. Calée aussi sur l'histoire individuelle.

II est ainsi possible de décrire trois stades évolutifs possibles de la consommation oenolique :

\section{Alcool convivial $=$ moderato cantabile}

Mettant de côté les pouvoirs anesthésique et antiseptique de l'éthanol, il peut être mentionné que l'alcool possède des vertus anxiolytique, euphorisante et désinhibitrice. Trois vertus qui conjuguées autorisent d'ailleurs plus facilement l'expression du désir. Ce dernier n'étant alors plus autant muselé par l'autocensure. II est ainsi dit que « le surmoi est soluble dans l'alcool ". L'alcool est donc ici plutôt facilitateur de l'expression du désir.

\section{Alcool médicament}

La boisson sert alors de remplissage pour calmer les souffrances du sujet. S'installe alors progressivement et insidieusement une dépendance psychologique. Le produit est là pour colmater la brèche existentielle. Ce que Bukowski appelle l'angoisse. Extrait de Mémoire d'un vieux dégueulasse [2] où on assiste à une scène dans laquelle deux protagonistes observent leur camarade blessé.

- « ouais, ouais...

- dis moi, Marty doit perdre tout son sang ?

- probablement

- et c'est de sang que l'on est fait?

- surtout....

- Comment surtout?

- II y a aussi les os et l'angoisse "

Dans le cas de l'alcool pris tel un médicament, la notion de désir commence à s'estomper au profit de celle de besoin. Et si le besoin de médicament se fait de plus en plus pressant, de plus en plus régulier, s'installe alors l'addiction.

\section{Alcool drogue}

La dépendance est omniprésente. Tout oscille entre le vide (manque) et le plein (" comme un outre " parfois). Tout tourne autour du produit. La toxicomanie s'inscrit. Le produit remplace la relation. Sexuellement, c'est le temps de l'impuissance et de la " frigidité ». Le désir sexuel est absent, enterré. Seule figure l'addiction.

Ainsi, faudrait-il nuancer ce que dit Shakespeare : « l'alcool augmente le désir, mais il diminue les performances ". De fait, l'alcool, pris de façon modérée et irrégulière augmente certes le désir, mais dans l'alcoolodépendance on observe la mort du désir au profit de l'addiction.

En couple, l'homme alcoolique reproduit presque invariablement le même schéma comportemental. Celui du couple Mère-Enfant dans des registres bipolaires : enfant sage / enfant alcoolisé, persécuté / persécuteur, alcoolophobe / alcoolophile... Les difficultés pour le couple apparaissent bien souvent dès le passage de l'état fusionnel à la relation d'amour durable. L'abandon de la fusion, faisant progressi- 
vement place à l'altérité et l'individuation, sera vécu comme un véritable traumatisme par l'alcoolique pour lequel l'autre n'existe essentiellement qu'en tant que pansement ou remplissage de sa faille, de son vide, de son trou. L'émergence du désir de l'autre sera subi par lui comme totalement destructurante. Dans ce couple Mère / Enfant [10], le " parent " va s'occuper de l'enfant alcoolisé. II n'est donc pas considéré comme sujet désirant par l'alcoolique. Si par malheur ce SUJET devient désirant, le buveur se heurte alors au désir de l'autre. Ce qui l'expose en retour, à l'insatisfaction, la frustration dont la seule réponse apaisante est pour lui l'alcool, principal (ou unique) OBJET de sa satisfaction.

L'addiction, dont l'alcoolisme n'est qu'une forme, serait donc une relation à l'OBJET (produit ou conduite). Le désir lui serait avant tout tourné vers le SUJET.

Du reste, après la période difficile du sevrage biologique s'ouvre le champ du désir de l'ancien buveur. Devenu abstinent il vit un authentique regain d'élan vital. L'émergence de son désir, le fait de (re)devenir sujet désirant sont décrits comme de véritables "shoot au moi ". Le processus addictif ne fait alors plus écran au désir du sujet. Seul hic (!) pour le couple, se produit alors la facétieuse rencontre de deux sujets désirants et... donc de leur altérité.

\section{L'HOMME DÉFONCÉ}

Si Bukowski a été une figure littéraire emblématique de l'alcool, c'est à Burroughs que revient celle de la défonce. Burroughs dans Junky [3] fait une description du vécu, des sensations et des réflexions sur sa conduite addictive. De façon fulgurante, en une phrase, il dépeint le parcours du camé. "On n'a pas l'intention de le devenir. On ne se réveille pas un matin en décidant d'être drogué...Un matin, on se réveille malade $»$.

L'expérience toxicomaniaque commence donc par un nondésir.

Dans les premiers temps de la rencontre avec le produit, tout drogué est d'abord un expérimentateur de la chimie du bonheur. Et bien vite, au delà de l'extase recherchée dans la prise du produit, le consommateur découvre d'autres vertus lénifiantes. Freud a du reste, à travers son expérience de la cocaïne, qualifié ces produits de " briseurs de soucis ". La toxicomanie s'inaugure donc par une " période rose " comme le dit Christiane F., une période où le sujet peut enfin voir la vie en rose. L'usage du psychotrope permet d'éluder les problèmes, de gommer les soucis. II met le sujet sur la touche, hors du temps et des ennuis ; le réconciliant avec les gens et les choses. Bref, s'il est "junky", il plane....s'il est alcoolique, il cuve. Ce gommage, cette annihilation des soucis par une substance est somme toute le rêve de tout un chacun et déjà évoqué comme le pharmakon nepentes dans l'lliade d'Homère.

"Briseur de souci » disait Freud [8]. Or, qu'est ce que le souci, sinon un désir réfréné, inassouvi, frustré, refoulé ? Dans un premier temps, donc, le sujet addict aurait trouvé la solution pour faire taire ses désirs insatisfaits.
Hélas, la période rose est éphémère et cède sa place à l'anthracite de la dépendance. Le sujet devient " accro » à sa dope. Il entre dans l'ère du besoin.

Ce besoin, la philosophe Guilia Sissa le qualifie de désir insatiable [12]. Elle décrit dans les lignes suivantes ce que l'on pourrait qualifier de détournement de la dialectique plaisir-manque chez le sujet addict. " Je définis la toxicomanie comme une pratique qui fait fonctionner réellement la puissance d'un désir devenu insatiable et de plus en plus dévorant, au point que la satisfaction jamais définitive se change ici en tolérance et en dépendance : fixation sur des produits dont on ne peut plus se passer, pour ne pas trop souffrir....C'est ainsi que le plaisir se trouve transformé : cessation de cette peine, non-douleur, plaisir négatif. "....Un matin, il se retrouve malade, disait Burroughs. Son plaisir est devenu négatif, il ne sert plus qu'à compenser un vide et non pas à créer un agrément supplémentaire.

Le toxicomane, comme l'alcoolique avec sa dialectique plein-vide serait donc l'illustration la plus flagrante de ce que dit Platon du désir. II reprendrait à son propre compte, en l'incarnant dans son expérience personnelle la métaphore du philosophe grec. A savoir la jarre fêlée comme représentation du désir et de son insatiabilité. On a beau la remplir, elle se vide tout autant. L'addiction, selon Guilia Sissa serait donc une métaphore amplifiée, exacerbée du désir platonicien. Le toxicomane qui se dit "défoncé " ne fait que crier qu'il est sans fond, dé-foncé, que son désirbesoin est sans fond, qu'il est insatiable. Tout comme ses cellules, ses neurones sont devenus insatiables vis-à-vis de l'apport d'une substance qui anesthésie l'intolérable souffrance du manque.

\section{PLAISIRS D'AMOUR}

Les neurobiologistes de leur côté expliquent l'installation du manque et de la dépendance par la théorie des processus opposants de Solomon. Cette théorie dit que lorsqu'un stimulus entraîne un état plaisant, il s'installe un contre-stimulus déplaisant juste après. Ainsi, comme le décrit Lucy Vincent dans Comment devient-on amoureux ? : «...si on répète plusieurs fois le même stimulus, une tolérance s'installe, ce qui signifie que les récepteurs à endorphines se fatiguent et produisent de moins en moins de plaisir. On pourrait s'attendre à ce que l'après-réaction diminue également. Et bien, c'est le contraire qui arrive : la répétition provoque un affaiblissement de la réaction agréable et une augmentation de l'après-réaction, d'où la sensation de manque... qui pousse à rechercher de plus en plus de stimulus plaisant...ainsi se crée la dépendance » [13].

Plus loin, dans son ouvrage, elle invite aussi à comparer les processus neuro-chimiques qui opèrent dans la toxicomanie et dans le processus amoureux. Que ce soit pour l'amour, ou pour l'héroïne, le même circuit est en jeu : celui de la récompense avec sa clef de voûte le noyau accumbens. Les circuits de la récompense fonctionnent principalement avec deux neurotransmetteurs : la dopamine et les endorphines. La dopamine responsable de la motivation, du désir 
et les endorphines à l'origine des sensations de plaisir. Pour elle, « l'état amoureux implique directement l'activité des neurones dans ces systèmes ". Ainsi, la dopamine serait la " dope de l'amour " et les endorphines le concrétiseraient dans un bain de bonheur.

La phrase " tu me manques " prendrait par là-même une interprétation biologique possible.

De là à envisager l'amour comme une forme d'addiction... ? (Figure 1).

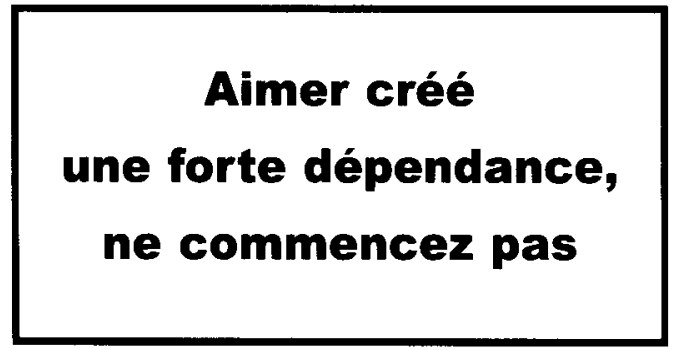

Figure 1 : Avertissement sanitaire.

Le philosophe André Comte-Sponville nous offre une possibilité de réponse en comparant Platon et Spinoza quant à leur discours sur l'amour [1]. Pour Platon, dans Le banquet, citant Socrate, il dit que l'amour est désir et le désir est manque. « Ce qu'on n'a pas, ce qu'on n'est pas, ce dont on manque, voilà les objets du désir et de l'amour ». A l'inverse, Aristote écrit : "aimer c'est se réjouir ". Ce que reprend Spinoza pour qui l'amour et le désir ne sont pas manque mais puissance et joie.

Ainsi, Platon aurait une vision addictive de l'amour et Spinoza une vision désirante de l'amour.

Reste à envisager si le sujet addict n'est pas une personne en manque de se réjouir, de jouir... L'homme frigorifié, l'homme défoncé comme des métaphores du « peine à jouir ". Des êtres en attente d'accéder au désir au sens spinozien. En attente de se muer en sujets désirants. Désir comme définissant le sujet : je désire donc j'existe.

Puisque le sujet non désirant est un être symboliquement mort. Un être qui n'est plus... un être presque au fond du TROU.

\section{REFERENCES}

1. BRENOT P., COMTE-SPONVILLE A., COSTA P. : Qu'est ce que l'amour ? Paris, L'esprit du temps, 2001.

2. BUKOWSKI C. : Journal d'un vieux dégueulasse. Paris, Les Humanoïdes associés, 1975.

3. BURROUGHS W.S. : Junky. Penguin Book, 1977. pour la traduction française : Paris, Belfond, 1979.

4. CHEBEL M. : Du désir. Paris, Payot, 2000.

5. CYRULNIK B. : Un merveilleux malheur. Paris, Odile Jacob, 1999.
6. DE MIJOLLA, SHENTOUB S.A. : Pour une psychanalyse de l'alcoolisme. Paris, Payot, 1973.

7. Mc DOUGALL J. : Eros aux mille et un visages. Paris, NRF, Ed. Gallimard, 1995.

8. MIRASHI R. : Désir et besoin. Paris, Ellipses Edition, 2001.

9. PASINI W. : La force du désir. Paris, Odile Jacob, 1993.

10. POLLOCK J. : Addiction et dépendance. Revue Française de Psychanalyse, 2004, 68 : 560-577.

11. REY P. : Le désir. Paris, Plon, 1999.

12. SISSA G. : Le plaisir et le mal. Paris, Odile Jacob, 1997.

13. VINCENT L. : Comment devient-on amoureux ? Paris, Odile Jacob, 2004

Communication au $X X 1^{\circ}$ Congrès de la Société d'Andrologie de Langue Française, Clermont-Ferrand, 9-11 Décembre 2004.

Manuscrit reçu : mai 2005 ; accepté juin 2005.

\author{
ABSTRACT \\ Desire and Addiction
}

Pascal COLAS

Analysis of the etymology of the two terms, desire and addiction, requires a distinction between their common aspects and their differences. Desire is an awareness of a need to obtain a known or imaginary object. Addiction is a derived from the Latin word addictere meaning slavery, alienation, physically constrained. The immobilization observed in the alienation inherent to addiction contrasts with the dynamic tendency associated with desire. Desire could be defined as an active force, while addiction is a passive force. Desire is an emanation of life, and addiction is an expression of a process of death. The desire of love can also be distinguished from dependent love (addiction), and the sentence "I miss you" reflects the addictive dimension of love.

Key words: alcohol, addiction, drug addiction, desire, love, alexithymia 